\title{
HIERARCHIES IN RECURSIVE FUNCTION THEORY $\left.{ }^{(}\right)$
}

\author{
BY \\ H. B. ENDERTON
}

1. Introduction. Hierarchies have been constructed as attempts to organize some collection of sets of integers according to degree of unsolvability. In this paper we will be concerned with transfinite hierarchies, the foremost example of which is the hyperarithmetic hierarchy $[\mathrm{D}$, p. 191, Definition 5.1], [K2, p. 200, the predicates $\left.H_{y}\right]$, [S1, p. 155 "Davis-Kleene hierarchy"]. In particular we are interested in conditions under which a pathological type of collapse can be avoided.

Let $N$ be the set of all non-negative integers: $0,1,2, \cdots$. This is the simplest infinite set, and we may justifiably claim to have a fair understanding of it. By contrast the power set $\mathscr{P} N$ of $N$ has uncountably many members, most of which cannot even be specifically named. The phrase "all subsets" does not help us comprehend the various sorts of subsets which may arise. (Since there is a natural correspondence between the infinite subsets of $N$ and the interval $(0,1]$, we could also formulate the problem in terms of comprehending the set of real numbers.)

By means of hierarchies we can organize large (but countable) subclasses of $\mathscr{P} N$. In effect we bring civilization to the fringe of the jungle $\mathscr{P} N$.

The basic principle of hierarchies can be stated, "Little steps for little feet." We choose a method of extending slightly any subclass of $\mathscr{P} N$ that we claim to have already tamed. Then starting (for example) with the class of recursive subsets of $N$, we apply our extension method over and over. With patience and fortitude we thus construct a transfinite hierarchy. The quality of the hierarchy depends on (i) the choice of the extension method and (ii) the method of achieving transfinite iteration.

In the following sections we will see concrete examples of the process we have described above in vague terms. Unfortunately there are complications which arise in the course of making matters precise. Meeting these complications requires a compromise between the dictates of expediency and motivation. For reasons of expediency, we will consider transfinite sequences (and more general arrays) of specific subsets of $N$. If we replace each such subset $A$ of $N$ by the class of all sets recursive in $A$, we obtain an expanding sequence of subclasses of $\mathscr{P} N$.

2. The stumbling-block. One approach is to construct a hierarchy of Turing degrees. Suppose we have (corresponding to the extension method) a function $F$

Received by the editors February 28, 1963.

(1) These results are contained in the author's dissertation [E] presented to the Department of Mathematics, Harvard University. 
defined on the set of degrees, and such that for any degree $a, F(a)$ is slightly higher than $\boldsymbol{a}$. (An example is the ordinary jump, defined by $F(\boldsymbol{a})=\boldsymbol{a}^{\prime}$ in the notation of $[\mathrm{KP}$, p. 384].) Then we can define an increasing function $G$ on the finite ordinals:

$$
\begin{aligned}
G(0) & =\text { the recursive degree, } \\
G(\alpha+1) & =F(G(\alpha)) .
\end{aligned}
$$

But the appropriate definition of $G(\omega)$ is a difficult problem. We might let $G(\omega)$ be the least upper bound of the $G(\beta)$ for $\beta<\omega$, if this least upper bound existed. But by a theorem of Spector [S2, p. 585] it does not; indeed no infinite increasing sequence of degrees has a least upper bound.

The method which has been used to meet this difficulty involves (1) using well-orderings or at least partial well-orderings of integers in place of ordinal numbers, and (2) using particular sets of integers in place of degrees. This requires making some artificial choices, but it enables us to get around the stumbling-block at limit ordinals. The purpose of this paper is to establish conditions under which the resulting hierarchy does not depend in any essential way on the artificial choices made.

3. Hierarchies. If $e$ is a Gödel number of $A$ from $B$ (i.e., $\{e\}^{B}$ is the characteristic function of $A$ ), then we will write

$$
A \leqq[e] B .
$$

Unless otherwise specified, "set" will mean "set of non-negative integers."

First we want to define the notion of a jump operation. The two most common examples are

(1) The ordinary jump oj:

$$
o j(A)=A^{\prime}=\left\{x:(\exists y) T_{1}^{A}(x, x, y)\right\} .
$$

(2) The hyperjump $h$ :

$$
h(A)=\left\{x:(\forall \alpha)(\exists y) T_{1}^{\alpha, A}(x, x, y)\right\} .
$$

The hyperjump is stronger than the ordinary jump in the following sense:

Definition. If $j$ and $k$ are two maps from $\mathscr{P} N$ into itself, then $k$ is as strong as $j$ iff there exists an integer $e$ such that for any set $A$

$$
j(A) \leqq[e] k(A) \text {. }
$$

Definition. A jump operation is a function $j$ from $\mathscr{P} N$ into itself such that

(1) there is a partial recursive function $J$ such that

$$
A \leqq[e] B \Rightarrow j(A) \leqq[J(e)] j(B) ;
$$

(2) $j$ is as strong as the ordinary jump oj.

A partial well-ordering $<_{1}$ is a transitive binary relation such that any nonempty set has a $<_{1}$-minimal element. The field $D$ of $<_{1}$ is 


$$
\left\{x:(\exists y)\left(x<_{1} y \text { or } y<{ }_{1} x\right)\right\} .
$$

It is helpful to define

$$
x \leqq_{1} y \Leftrightarrow_{\mathrm{df}} x<_{1} y \text { or }(x=y \& x \in D) .
$$

There is a unique function $\mid$ from $D$ into the ordinals defined inductively:

$$
\begin{gathered}
|x|=\text { the least ordinal } \beta \text { such that } \\
\qquad\left(\forall y<_{1} x\right)|y|<\beta .
\end{gathered}
$$

THEOREM 1. If $\beta<|x|$ then $\left(\exists y<_{1} x\right)|y|=\beta$.

If a partial well-ordering is to be the basis for a hierarchy, then we would like it to be well-behaved. But if we make very strong demands (e.g., the $r$-systems of [K1]) then we place an upper bound on the possible values of $|x|$ for $x$ in $D$. The following definition provides some regularity without imposing any such upper bounds.

Definition. A system of notations is a partial well-ordering $<_{1}$ which satisfies

(i) If $b \in D$ and $|b|=0$, then $b=1$.

(ii) Each $b$ in $D$ has a unique successor, which is $2^{b}$.

(iii) If $|b|$ is a limit ordinal then $b \neq 2^{(b)}$.

These conditions could be replaced by something less specific, which would simply assert the existence of some partial recursive functions with certain properties (cf. Definition 1 of [W]). But there seems to be little motivation for generalized versions. And the notation is simplified by the special form of our definition. In particular if $x \neq 0$ then we define

$$
x^{*}=2^{x}
$$

(Leave $0^{*}$ undefined.) Then for $x$ in $D$ we have

$$
\left|x^{*}\right|=|x|+1
$$

DefinItION. A hierarchy is an ordered pair $\left\langle j,<_{1}\right\rangle$ consisting of a jump and a system of notations.

EXAMPLE. The hyperarithmetic hierarchy is $\left\langle o j,<_{0}\right\rangle$, where $<_{0}$ is the ordering defined in $[\mathrm{K} 1, \mathrm{p} .155]$ and in [KR, Theorem 2].

For the hierarchy $\left\langle j,<_{1}\right\rangle$ we define inductively a function $H$ from $D$ into PN:

(1) $H(1)=N$.

(2) $H\left(b^{*}\right)=j(H(b)), \quad b$ in $D$.

(3) If $|b|$ is a limit ordinal then

$$
\begin{aligned}
H(b) & =\left\{\langle x, y\rangle: y<<_{1} b \& x \in H(y)\right\} \\
& =\bigcup_{y<1 b}(H(y) \times\{y\}) .
\end{aligned}
$$


Here ordered pair and cartesian product are to be formed by means of the pairing function

$$
\langle x, y\rangle=2^{x} 3^{y}
$$

Cases (1) and (2) of the definition of $H$ are intuitively natural. (For a hierarchy relativized to a set $A$ we would want $H(1)=A$.) Case (3) is analogous to the definition of recursive join in [KP, p. 392]. Observe that if $|b|$ is a limit ordinal and $y<_{1} b$, then $H(y)$ is recursive in $H(b)$. In fact the reduction is uniform in $y$ :

$$
x \in H(y) \Leftrightarrow\langle x, y\rangle \in H(b)
$$

We hope that $H(b)$ is as simple as possible, given that the above reducibility property should hold. We shall see that in certain circumstances there is some basis for this hope.

Since our construction of the hyperarithmetic hierarchy differs from those in the literature, we state the equivalence.

THEOREM 2. Consider the hyperarithmetic hierarchy $\left\langle o j,<_{0}\right\rangle$. For each $b$ in the field 0 of $<_{0}$, our set $H(b)$ has the same degree of unsolvability as the predicate $H_{b}$ defined in [K2]. Furthermore the equivalence is uniform in $b$.

The proof is an application of the recursion lemma stated in $\$ 4$. Details can be found in [E].

Recall that we used a partial well-ordering $<_{1}$ in the construction of the hierarchy because we were unable to use the classical ordinals. Our interest lies in iterating $j$, not in examining $<_{1}$. But this is not to say that different choices of $<_{1}$ will lead to equivalent results. There are hierarchies in which the well-ordering is not content with just keeping order, but usurps power itself. For example given any set $S$ we can form the relativized ordering $<_{0}^{S}$ on $0^{S}$ [K2, p. 209]. The correct way to construct a relativized hierarchy is to use $<_{0}^{s}$ and define $H(1)=S$. (Other cases of the definition of $H$ are as before.) But if we instead apply our definitions as they stand to the ordering $<{ }_{0}^{S}$, then for any jump $j$ the hierarchy $\left\langle j,<_{0}^{S}\right\rangle$ has the following undesirable feature:

THEOREM 3. There is $a b$ in $0^{S}$ such that $|b|=\omega^{2}$ and $S$ is recursive in $H(b)$.

The proof will be found in $\$ 4$.

If the degree of $S$ is very high, then it is clear that this represents a failure of the hierarchy to serve its purpose. This collapse of the hierarchy is due to the fact that we have used an ordering which is too high-powered for the jump. We want to consider circumstances in which this type of collapse can be avoided. Put another way, what relationship between the jump and the ordering will assure that the degrees of the sets $H(a)$ are not artificially elevated?

One method for making this problem precise is to investigate the property of uniqueness. We say that uniqueness holds in a hierarchy if for any $a$ and $b$ in the 
field of $<_{1},|a|=|b|$ implies $H(a)$ and $H(b)$ are recursive in each other (i.e., Turing equivalent). This condition fails in our example above with $<_{0}^{S}$. Uniqueness implies that different paths through $<_{1}$ lead to the same Turing degrees. This is certainly a desirable property, but we would like something even stronger. For example if the ordering is linear, then uniqueness automatically holds, but it gives no comfort.

A stronger property would be the following: If $<_{1}$ is replaced by another ordering $<_{2}$, then the degrees obtained (at any particular ordinal) are at least as high as before. This will imply of course that uniqueness holds.

In order to enable us to treat two orderings at once we now modify our notation. Now $<_{1}$ will have field $D_{1}$. The ordinal associated with a member $b$ of $D_{1}$ will be $|b|_{1}$. The function from $D_{1}$ into $\mathscr{P}(N)$ will be $H_{1}$. For the ordering $<_{2}$ we will have correspondingly $D_{2},|s|_{2}$, and $H_{2}$. Always there will be just one jump $j$ for both orderings. Now we can give a precise statement of the desirable property described.

Definition. A system $<_{1}$ of notations is canonical for a jump $j$ iff given any system $<_{2}$ of notations, there is a partial recursive function $\phi$ such that

$$
|a|_{1} \leqq|s|_{2} \Rightarrow H_{1}(a) \leqq[\phi(a, s)] H_{2}(s) \text {. }
$$

We will also say that the hierarchy $\left\langle j,<_{1}\right\rangle$ is minimal. We can now state our central theorem:

THEOREM 4. A sufficient condition for a system $<_{1}$ of notations to be canonical for $j$ is the following:

(*) There exist partial recursive functions $F$ and $G$ such that whenever $|b|_{1}$ is not zero then $F(b)<_{1} b$ and $\left\{y: y<_{1} b\right\}$ is recursive in $H_{1}(F(b))$ with Gödel number $G(b)$.

Intuitively, condition ( $*$ ) says that the set of all predecessors of a point $b$ is recursive in an earlier $H$-set, and in a uniform manner. The proof of the theorem will be in $\$ 4$; applications and related results will be discussed in $\S 5$. The following is an immediate consequence of ( $\mathbf{k})$ :

THEOREM 5. Suppose $<_{1}$ and $j$ satisfy $(*)$, and let $|b|_{1}$ be a limit ordinal. If $A$ is any set such that

$$
y<_{1} b \Rightarrow H(y) \text { recursive in } A \text {, uniformly in } y
$$

then $H_{1}(b)$ is recursive in $A$.

4. Proofs. In a system of notations the only element $b$ with $|b|$ equal to a finite integer $n$ is $b=\bar{n}$, where

$$
\begin{aligned}
\overline{0} & =1, \\
\overline{n+1} & =\bar{n}^{*} .
\end{aligned}
$$


Proof of Theorem 3. We may suppose $S$ is infinite; let its members be (in ascending order) $s_{0}, s_{1}, \cdots$. Let $F$ be a recursive function such that

(a) $F(x, y)$ is a Gödel number of the partial function:

$$
\begin{aligned}
\{F(x, y)\}(1) & =x, \\
\{F(x, y)\}\left(n^{*}\right) & =\{F(x, y)\}(n)^{*} .
\end{aligned}
$$

(b) $F(x, y)$ encodes (in any uniformly retrievable manner) the integer $y$. Now let

$$
\begin{aligned}
G(1) & =1, \\
G\left(\bar{n}^{*}\right) & =3 \cdot 5^{F\left(G(\bar{n}) .\left\langle s_{n}, n\right\rangle\right)} .
\end{aligned}
$$

Then $G$ is partial recursive in $S$; let $e$ be a Gödel number of $G$ from $S$ and let $b=3 \cdot 5^{e}$.

Now $G(\bar{n}) \in O \subseteq O^{S}$ and $|G(\bar{n})|=\omega \cdot n$. Thus $b$ is in $O^{S}$ and $|b|=\omega^{2}$.

From $H(b)$ we can enumerate

$$
\left\{y:(\exists x)\left\langle x, 3 \cdot 5^{y}\right\rangle \in H(b)\right\} .
$$

Each $y$ in this set is of the form

$$
y=F\left(G(\bar{n}),\left\langle s_{n}, n\right\rangle\right),
$$

whence we can compute $n$ and $s_{n}$. Thus we can enumerate from $H(b)$ the set $S$ in ascending order.

The above result is analogous to XIII in [K2]. The lemma below is a basic tool in studies of hierarchies. It is adapted from [R].

RECURSION LEMMA 6. Let $<_{1}$ be a partial well-ordering of a set $D$. Let $P$ be a two-place predicate, and let $K$ be a partial recursive function such that for any $b$ in $D$ and any $q$ :

$$
\left(\forall y<_{1} b\right) P(\{q\}(y) ; y) \Rightarrow P(K(q, b) ; b) .
$$

Then we can find a partial recursivefunction $F$ such that for all $b$ in $D, P(F(b) ; b)$.

This is the recursive function theory counterpart of transfinite induction. The hypothesis says that whenever you have a uniform way of making $P$ true for all $y$ less than $b$, then you can effectively find a way to make $P$ true of $b$. The conclusion states that you have a uniform method for making $P$ true of all members of $D$.

Proof of recursion lemma. By the recursion theorem [IM, p. 353] we can find an integer $e$ such that $\{e\}=\lambda x K(e, x)$. We claim that for all $b$ in $D, P(\{e\}(b) ; b)$. For otherwise there is a minimal $b$ in $D$ for which this fails. By the minimality

$$
\left(\forall y<{ }_{1} b\right) P(\{e\}(y) ; y) \text {, }
$$

whence by hypothesis 


$$
P(K(e, b) ; b) \text {, i.e., } P(\{e\}(b) ; b) \text {. }
$$

Contradiction. Thus we may take $F=\{e\}$.

In the following we will apply this lemma when $P$ has more variables, but the extension is trivial.

As an abbreviation define

$$
\operatorname{Lim}(u) \Leftrightarrow_{\mathrm{df}} u \neq 2^{(u)_{0}} .
$$

Instead of (for example)

$$
(\forall u)\left(u^{* *}<_{2} s \Rightarrow A\right)
$$

we will write simply

$$
\left(\forall u^{* *}<_{2} s\right) A
$$

Proof of Theorem 4. The proof will be informal. Recursive functions will be described from the Turing machine viewpoint, cf. [D]. First introduce an ordering $<_{x}$ on $D_{x}$, the cartesian product of $D_{1}$ and $D_{2}$.

$$
\langle b, t\rangle<_{x}\langle a, s\rangle \Leftrightarrow_{\mathrm{df}}\left(b \leqq_{1} a \text { and } t<{ }_{2} s\right) \text { or }\left(b<_{1} a \text { and } t \leqq_{2} s\right) \text {. }
$$

The proof will use the recursion lemma, i.e., effective transfinite induction. The induction hypothesis will consist of two parts. One will be concerned with the ability to recognize when the inequality

$$
|c|_{1} \leqq|u|_{2}
$$

holds; the other part will be more directly related to the desired conclusion. Define then:

$$
Q(q, a, s) \Leftrightarrow_{\mathrm{df}}\left(\forall c<_{1} a\right)\left(\forall u^{* *}<_{2} s\right)\left[\{q\}^{H_{2}(s)}(c, u) \text { halts } \Leftrightarrow|c|_{1} \leqq|u|_{2}\right] .
$$

This can be read " $q$ detects ordinal inequality from $H_{2}(s)$ below $\langle a, s\rangle$." Of course

$$
\{q\}^{B_{2}(s)}(c, u) \text { halts } \Leftrightarrow(\exists w) T_{2}^{H_{2}(s)}(q, c, u, w) .
$$

Next define

$$
\begin{aligned}
& P(r, q ; a, s) \Leftrightarrow_{\mathrm{df}} \\
& \quad[\operatorname{Lim}(s) \Rightarrow Q(q, a, s)] \text { and }\left[|a|_{1} \leqq|s|_{2} \Rightarrow H_{1}(a) \leqq[r] H_{2}(s)\right] .
\end{aligned}
$$

In order to apply the recursion lemma to $P$ we need partial recursive functions $K_{1}, K_{2}$ such that for $\langle a, s\rangle$ in $D_{x}$,

$$
\left(\forall\langle b, t\rangle<_{x}\langle a, s\rangle\right) P(\{r\}(b, t),\{q\}(b, t) ; b, t)
$$

implies 


$$
P\left(K_{1}(r, q, a, s), K_{2}(r, q, s) ; a, s\right) .
$$

Assume then that $\langle a, s\rangle \in D_{x}$ and the inductive hypothesis holds:

$$
\left(\forall\langle b, t\rangle<_{x}\langle a, s\rangle\right) P(\{r\}(b, t),\{q\}(b, t) ; b, t) .
$$

First we seek an integer

$$
\hat{r}=K_{1}(r, q, a, s)
$$

such that

$$
|a|_{1} \leqq|s|_{2} \Rightarrow H_{1}(a) \leqq[\hat{r}] H_{2}(s) .
$$

Our construction of $\hat{r}$ will use the integer

$$
\hat{q}=K_{2}(q, r, s)
$$

so that logically the latter should come first. But the $r$-half will show why one needs the $q$-half at all.

Assume then that $|a|_{1} \leqq|s|_{2}$. Then $\hat{r}$ must be the Gödel number of a Turing machine (also called $\hat{r}$ ) such that $\{\hat{r}\}^{H_{2}(s)}$ is the characteristic function of $H_{1}(a)$. The behavior of $\hat{r}$ will be described by cases, all effectively recognizable.

Case I. $a=1$ or $s=1$. Trivial.

Case II. $a=b^{*}$ and $s=t^{*}$, where $b=(a)_{0}, t=(s)_{0}$.

Then

$$
\begin{aligned}
|a|_{1} \leqq|s|_{2} & \Rightarrow|b|_{1} \leqq|t|_{2} \\
& \Rightarrow H_{1}(b) \leqq[\{r\}(b, t)] H_{2}(t) .
\end{aligned}
$$

So let

$$
\hat{r}=J(\{r\}(b, t)),
$$

where $J$ is the function mentioned in the definition of jump.

Case III. $\operatorname{Lim}(a)$. For each $\langle x, y\rangle$ we want

$$
\{\hat{r}\}^{H_{2}(s)}(\langle x, y\rangle)= \begin{cases}1 & \text { if } y<_{1} a \text { and } x \in H_{1}(y), \\ 0 & \text { otherwise. }\end{cases}
$$

By (*)

$$
\left\{y: y<_{1} a\right\} \leqq[G(a)] H_{1}(F(a)),
$$

where $F(a)<_{1} a$. And by inductive hypothesis

$$
H_{1}(F(a)) \leqq[\{r\}(F(a), s)] H_{2}(s) .
$$

Hence $\hat{r}$ can begin by determining whether or not $y<_{1} a$. If so, then

$$
H_{1}(y) \leqq[\{r\}(y, s)] H_{2}(s) \text {. }
$$


Thus $\hat{r}$ can determine whether or not $x \in H_{1}(y)$. This description defines $\hat{r}$ effectively as a function of $a, s$, and $r$.

Case IV. Lim (s) and $a=b^{*}$, where $b=(a)_{0}$. This is the only difficult case. If $\hat{r}$ can locate an integer $t$ such that

$$
t^{*}<_{2} s \text { and }|b|_{1} \leqq|t|_{2},
$$

then we will have

$$
H_{1}(a) \leqq\left[\{r\}\left(a, t^{*}\right)\right] H_{2}\left(t^{*}\right)
$$

and

$$
x \in H_{2}\left(t^{*}\right) \Leftrightarrow\left\langle x, t^{*}\right\rangle \in H_{2}(s)
$$

Thus it remains only to describe how $\hat{r}$ can find $t$.

The answer is provided by $\hat{q}$. The machine $\hat{r}$ enumerates (from $\mathrm{H}_{2}(s)$ ) the various $t$ such that $t^{* *}<_{2} s$, and for each begins the computation of

$$
\{\hat{q}\}^{H_{2}(s)}(b, t) \text {. }
$$

Since $Q(\hat{q}, a, s)$ holds, the above computation halts iff $|b|_{1} \leqq|t|_{2}$. Eventually this must occur, so eventually $\hat{r}$ will discover a suitable value for $t$.

This completes the description of

$$
\hat{r}=K_{2}(q, r, s),
$$

but we still need an integer

$$
\hat{q}=K_{2}(q, r, s)
$$

such that whenever $\operatorname{Lim}(s)$ then

$$
Q(\hat{q}, a, s),
$$

i.e., $\hat{q}$ detects ordinal inequality from $\mathrm{H}_{2}(s)$ below $\langle a, s\rangle$. Suppose then that $\operatorname{Lim}(s)$ and we are given $c$ and $u$ such that $c<_{1} a$ and $u^{* *}<_{2} s$. Then $\hat{q}$ must be the Gödel number of a Turing machine (also called $\hat{q}$ ) such that

$$
\{\hat{q}\}^{H_{2}(s)}(c, u) \text { halts } \Leftrightarrow|c|_{1} \leqq|u|_{2} .
$$

We will describe the steps made by this machine.

We may assume $\operatorname{Lim}(u)$, for otherwise $\hat{q}$ could replace $u$ by $\log _{2} u$ and $c$ by $\log _{2} c$ (if $\left.c=(c)_{0}^{*}\right)$ and iterate until the $\operatorname{Lim}(u)$ situation was reached. Next $\hat{q}$ computes $F(c)$ and $\{q\}(c, u)$.

By inductive hypothesis

$$
Q(\{q\}(c, u), c, u)
$$

i.e., 


$$
\left(\forall d<_{1} c\right)\left(\forall t^{* *}<_{2} u\right)\left(M(d, t) \text { halts } \Leftrightarrow|d|_{1} \leqq|t|_{2}\right)
$$

where

$$
M={ }_{\mathrm{df}}\{\{q\}(c, u)\}^{\mathrm{H}_{2}(u)}
$$

Next $q$ enumerates (from $H_{2}(s)$ ) the various $t$ such that $t^{* *}<_{2} u$, and for each begins the computation of $M(F(c), t)$. This is possible since

$$
t^{* *}<_{2} u \Leftrightarrow(\exists x)\left\langle\left\langle x, t^{* *}\right\rangle, u\right\rangle \in H_{2}(s) \text {. }
$$

By inductive hypothesis and (*)

$$
M(F(c), t) \text { halts iff }|F(c)|_{1} \leqq|t|_{2} .
$$

Now if $|c|_{1} \leqq|u|_{2}$ then (using $\operatorname{Lim}(u)$ ) for some $t^{* *}<_{2} u$ we will have $|F(c)|_{1} \leqq|t|_{2}$, whence $M(F(c), t)$ will halt. Thus if halting does not occur for any $t$, it follows that $|c|_{1}<|u|_{2}$.

And

$$
\{\hat{q}\}^{H_{2}(s)}(c, u)
$$

will not halt, so in this case $\hat{q}$ meets our requirements. On the other hand if for some $t, M(F(c), t)$ does halt, then

$$
|F(c)|_{1} \leqq|t|_{2}<|u|_{2}
$$

It is clear that

$$
\begin{aligned}
|c|_{1} \leqq|u|_{2} & \Leftrightarrow\left(\forall d<_{1} c\right)\left(\exists v^{* *}<_{2} u\right)|d|_{1} \leqq|v|_{2} \\
& \Leftrightarrow(\forall d)\left(d<_{1} c \Rightarrow(\exists v)\left(v^{* *}<_{2} u \text { and } M(d, v) \text { halts }\right)\right) .
\end{aligned}
$$

By (*)

$$
\left\{d: d<_{1} c\right\} \leqq[G(c)] H_{1}(F(c))
$$

and by inductive hypothesis

$$
H_{1}(F(c)) \leqq[\{r\}(F(c), u)] H_{2}(u) .
$$

Furthermore

$$
v^{* *}<_{2} u \Leftrightarrow(\exists x)\left\langle x, v^{* *}\right\rangle \in H_{2}(u)
$$

and

$$
M(d, v) \text { halts } \Leftrightarrow(\exists y) T_{2}^{B_{2}(u)}(\{q\}(c, u), d, v, y) .
$$

Hence we can effectively put the predicate

$$
|c|_{1} \leqq|u|_{2}
$$

in $\Pi_{2}^{H_{2}(u)}$ form. Since $j$ is as strong as $o j$, this yields a Gödel number of the predicate in 


$$
\mathrm{H}_{2}\left(u^{* *}\right)
$$

and thence a Gödel number in $H_{2}(s)$. So $\hat{q}$ concludes by testing to see if $|c|_{1} \leqq|u|_{2}$ (as described) and halting iff this inequality is true.

This completes the description of $K_{1}$ and $K_{2}$. We now apply the recursion lemma to obtain a partial recursive function $\phi$ such that

implies

$$
|a|_{1} \leqq|s|_{2}
$$

$$
H_{1}(a) \leqq[\phi(a, s)] H_{2}(s) .
$$

Observe that the function $\phi$ does not depend on $<_{2}$. Gödel numbers for $K_{1}$ and $K_{2}$ (and hence for $\phi$ ) can be found from Gödel numbers of $F, G, J$, and the function relating $j(A)$ to $o j(A)$.

Actually it is sufficient that $(*)$ hold only when $|b|_{1}$ is a limit ordinal. The $q$-half of the proof can be restricted to limit ordinals, with only notational complications.

5. Continuation. Let us first consider some applications of Theorem 4. If $j=o j$ and $<_{1}=<_{2}=<_{0}$, then we have the uniqueness theorem of Spector [S1, Theorem 5]. We can obtain an extension of Spector's theorem to Kleene's system $S_{1}$ of notations [K1] by taking

$$
<_{1}=<_{2}=R \cap\left(S_{1} \times S_{1}\right)
$$

where $R$ is the most natural recursively enumerable relation for which

$$
<_{0}=R \cap(O \times O) .
$$

This extension to $S_{1}$ was first obtained by Putnam [P]. In either case we obtain a stronger result by allowing $<_{2}$ to vary. But our theorem does not apply to $o j$ and Kreider's ordering $\mathscr{C}[\mathrm{KR} ; \mathrm{P}]$.

If we use the hyperjump $h$ then we can apply our theorem to a wider class of orderings. It is easy to see that $<_{0}^{o}$ is canonical for $h$. In fact $<_{0}^{s}$ is canonical for $h$ for any of the following choices of $S$ :

$$
o, O^{o}, O^{\left(o^{o}\right)}, \cdots \text {. }
$$

Let us return for a moment to the difficulties we encountered in $\$ 2$. There we tried to construct a function $G$ from the ordinals into the set of degrees. Let us try again. Instead of the function $F$ used in $\$ 2$ we use the function on degrees induced by a jump $j$. (This induced function is also called $j$.)

$$
\begin{aligned}
G(0) & =\text { the recursive degree, } \\
G(\alpha+1) & =j(G(\alpha)) .
\end{aligned}
$$

If $\lambda$ is a limit ordinal then $G(\lambda)$ is defined iff there exists a system $<_{1}$ of notations 
which is canonical for $j$ and whose field contains a $b$ with $|b|=\lambda$. In this case we let

$$
G(\lambda)=\text { degree of } H(b) .
$$

By our theorem this depends neither on the choice of $<_{1}$ nor on the choice of $b$.

This function $G$ has the pleasing feature of having been defined by the jump $j$ alone. The definition is, however, rather unwieldy. It would be convenient if we could construct, given $j$, a system $<_{j}$ of notations which would be canonical for $j$ and such that the ordinal

$$
\left|<_{j}\right|={ }_{\mathrm{df}} \text { l.u.b. }|b|, \quad b \in D_{j},
$$

is as large as possible. As an approximation to this goal we now construct a maximal system of notations $<_{j}$ such that the hierarchy $\left\langle j,<_{j}\right\rangle$ satisfies $(*)$.

The basic idea is to construct the ordering and the hierarchy simultaneously. At limit points we insert into the ordering everything that will not violate ( $*$ ).

Define by induction the set $L(\alpha)$ for each ordinal number $\alpha$ :

$$
\begin{aligned}
L(0) & =\{\langle 1,2\rangle\}, \\
L(\alpha+1)=L(\alpha) & \cup\left\{\left\langle x, x^{*}\right\rangle:\langle 1, x\rangle \in L(\alpha)\right\} \\
& \cup\{\langle x, y\rangle:(\exists t)(\langle x, t\rangle \in L(\alpha) \text { and }\langle t, y\rangle \in L(\alpha))\} .
\end{aligned}
$$

If $\lambda$ is a limit ordinal, then before defining $L(\lambda)$ we first let

$$
L_{\lambda}=\bigcup_{\alpha<\lambda} L(\lambda)
$$

Then $L_{\lambda}$ is a system of notations; form the hierarchy $\left\langle j, L_{\lambda}\right\rangle$. Then we can define $L(\lambda)=L_{\lambda} \cup\left\{\left\langle t, 3^{b} 5^{e}\right\rangle: b\right.$ is in the field of $L_{\lambda}$ and $e$ is a Gödel number from $H(b)$ of a subset $S$ of the field of $L_{\lambda}$ such that

(i) $b \in S$,

(ii) $(\forall x \in S)(\exists y \in S)|x|<|y|$,

(iii) $(\forall x \in S)(\forall y)\left(\langle y, x\rangle \in L_{\lambda} \Rightarrow y \in S\right)$ and $t \in S$.

Now let

and let $D_{j}$ be the field of $<_{j}$.

$$
<_{j}=\bigcup_{a} L(\alpha)
$$

Then $<_{j}$ is a system of notations. If $3^{b} 5^{e} \in D_{j}$, then

$$
\left\{t: t<{ }_{j} 3^{b} 5^{e}\right\}
$$

is recursive in $H(b)$ with Gödel number $e$. Hence $\left\langle j,<_{j}\right\rangle$ satisfies ( $*$ ).

We claim that $<_{j}$ is maximal in the following sense: Let $<_{1}$ be any system of notations such that $\left\langle j,<_{1}\right\rangle$ satisfies $(*)$. Then we will construct a partial recursive map $M$ from the field $D_{1}$ of $<_{1}$ into $D_{j}$ which preserves ordinals, i.e., 


$$
\left(\forall x \in D_{1}\right)|M(x)|_{j}=|x|_{1} .
$$

(Again we have two orderings, and so use subscripts liberally.)

We may suppose that

$$
x<_{1} y \text { and } \operatorname{Lim}(y) \Rightarrow x^{*}<_{1} y .
$$

For otherwise we could extend $<_{1}$ to make this true without loss of ( $*$ ) and without changing $|t|_{1}$ for any $t$ in $D_{1}$. By Theorem 4 there is a partial recursive function $Q$ such that

$$
\begin{aligned}
& s \in D_{1} \text { and } b \in D_{j} \text { and } \\
& \qquad|s|_{1} \leqq\left.\left.\right|^{*} b\right|_{j} \Rightarrow H_{1}(s) \leqq[Q(s, b)] H_{j}(b) .
\end{aligned}
$$

We will construct $M$ by using the recursion theorem, along the lines of the lemma in [R]. The property of $M$ we want is that for each $s$ in $D_{1}$ the following hold:

(a) $M(s)$ is defined and is a member of $D_{j}$.

(b) $|M(s)|_{j}=|s|_{1}$.

(c) $\left(\forall b<_{j} M(s)\right)\left(\exists t<_{1} s\right) M(t)=b$.

The idea of the construction is this: Suppose we have an initial approximation to $M$ which satisfies (a)-(c) for all $s$ such that $s<_{1} u$. We want to define $M(u)$. In the case $\operatorname{Lim}(u)$, we map

$$
\left\{t: t<{ }_{1} u\right\}
$$

by the approximation into $D_{j}$, thereby obtaining a subset $S$ of $D_{j}$ which will be recursive in $H_{j}\left(M(F(u))^{*}\right)$. ( $F$ is the function of $(*)$ for $<_{1}$; we will also use the $G$ function.) This will lead to a member

$$
3^{M(F(u))^{*}} \cdot 5^{e}
$$

of $D_{j}$ which we will want to be $M(u)$.

First let $\lambda x x \circ y$ be a recursive function such that for any sets $A, B, C$

$$
A \leqq[x] B \text { and } B \leqq[y] C \Rightarrow A \leqq[x \circ y] C .
$$

Since $j$ is as strong as $o j$, there is a $k$ such that for any set $A, A^{\prime} \leqq[k] j(A)$. And the image of $A$ under $\{e\}$ :

$$
\{e\}(A)=_{\mathrm{df}}\left\{x:(\exists t)\left(t \in A \text { and }(\exists w)\left(T_{1}(e, t, w) \text { and } U(w)=x\right)\right)\right\}
$$

is recursively enumerable in $A$, uniformly in $e$. That is, there is a recursive function $N$ such that $N(e)$ is a Gödel number of $\{e\}(A)$ from $A^{\prime}$. Define now the partial recursive function $K$ : 


$$
\begin{aligned}
& K(e, x) \simeq\left\{\begin{array}{l}
1 \text { if } x=1, \\
\{e\}(y)^{*} \text { if } x=y^{*}, \\
\text { in all other cases: }
\end{array}\right.
\end{aligned}
$$

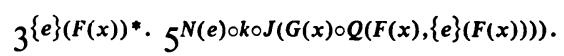

By the recursion theorem we can find an integer $e$ such that

Let $M=\{e\}$.

$$
\{e\}=\lambda x K(e, x) \text {. }
$$

We claim that for every $s$ in $D_{1}$, properties (a)-(c) hold. For otherwise there would be a minimal $s$ for which one of (a), (b), (c) failed.

Clearly $s \neq 1$. Furthermore we could not have $s=(s)_{0}^{*}$, by the minimality of $s$ and because

Hence Lim (s). Let

$$
d<_{j} b^{*} \Rightarrow d \leqq{ }_{j} b
$$

$$
\begin{aligned}
& d=M(F(s)), \\
& f=N(e) \circ k \circ J(G(s) \circ Q(F(s), d))
\end{aligned}
$$

so that $M(s)=3^{d^{*}} \cdot 5^{f}$. Let $S$ be the image under $M$ of

$$
\left\{t: t<_{1} s\right\} \text {. }
$$

Then $f$ exists and is a Gödel number of $S$ from $H_{j}\left(d^{*}\right)$. We further claim that

$$
\left\langle 1,3^{d^{*}} \cdot 5^{f}\right\rangle \in L\left(|s|_{1}\right) \text {. }
$$

Looking at the definition of $L\left(|s|_{1}\right)$ and putting $d^{*}$ in place of $b$, we see that (i) and (ii) hold. If $x \in S$ and $y<_{j} x$ then there is some $u<_{1} s$ such that $M(u)=x$. Applying (c) to $u$ we see that $y \in S$, whence (iii) holds.

Therefore (a) is true of $s$. Since

$$
\begin{aligned}
\left|3^{d^{*}} \cdot 5^{f}\right|_{j} & =\bigcup\left\{|c|_{j}: c \in S\right\} \\
& =\bigcup\left\{|t|_{1}: t<_{1} s\right\}=|s|_{1}
\end{aligned}
$$

(b) also holds. And (c) is obvious by our construction .

THeOREM 7. $<_{j}$ is a maximal system of notations such that $\left\langle j,<_{j}\right\rangle$ satisfies( $(*)$.

In the case of the ordinary jump oj we do not get outside the class of hyperarithmetic sets, and thus $\left\langle o j,<_{o j}\right\rangle$ is essentially the hyperarithmetic hierarchy. In the case of the hyperjump $h$ we obtain an ordering $<_{h}$ with field $D_{h}$.

THEOREM 8. For all $b$ in $D_{h}$,

$$
H_{h}(b) \in \Sigma_{2}^{1} \cap \Pi_{2}^{1} .
$$


The proof is a straightforward application of the recursion lemma; see [E]

6. Further problems. The ordinal number

$$
\left|<_{j}\right|=\bigcup\left\{|b|: b \in D_{j}\right\}
$$

$s$ a measure of the strength of Theorem 4 . We would like to compare this ordinal with

$$
\bigcup\left\{\left|<_{1}\right|:<_{1} \text { is canonical for } j\right\} \text {. }
$$

What is the relationship of these ordinals? In particular, does equality hold?

Suppose one wishes to iterate a jump over an ordering longer than any canonical ordering. The resulting hierarchy cannot be minimal, but it still may be "almost minimal." Recent work in this direction has been done by Luckham [L].

\section{BIBLIOGRAPHY}

[D] M. Davis, Computability and unsolvability, McGraw-Hill, New York, 1958.

[E] $\mathrm{H}$. Enderton, Hierarchies in recursive function theory, doctoral dissertation, Harvard University, 1962.

[IM] S. C. Kleene, Introduction to metamathematics, Van Nostrand, New York, 1952.

[K1] - On notation for ordinal numbers, J. Symbolic Logic 3 (1938), 150-155.

[K2] —-, Hierarchies of number-theoretic predicates, Bull. Amer. Math. Soc. 61 (1955), 193-213.

[K3] — Arithmetical predicates and function quantifiers, Trans. Amer. Math. Soc. 79 (1955), 312-340.

[KP] S. C. Kleene and E. L. Post, The upper semi-lattice of degrees of recursive unsolvability, Ann. of Math. (2) 59 (1954), 379-407.

[KR] D. Kreider and H. Rogers, Jr., Constructive versions of ordinal number classes, Trans. Amer. Math. Soc. 100 (1961), 325-369.

[L] D. Luckham, Topics in the theory of constructive hierarchies, doctoral dissertation, M.I.T., 1963.

[P] $\mathrm{H}$. Putnam, Uniqueness ordinals in higher constructive number classes, Essays on the Foundations of Mathematics, pp. 190-206, Magnes Press, Jerusalem, 1961.

[R] H. Rogers, Jr., Recursive functions over well ordered partial orderings, Proc. Amer. Math. Soc. 10 (1959), 847-853.

[S1] C. Spector, Recursive well-orderings, J. Symbolic Logic 20 (1955), 151-163.

[S2] - On degrees of recursive unsolvability, Ann. of Math. (2) 64 (1956), 581-592.

[W] H. Wang, Ordinal numbers and predicative set theory, Z. Math. Logik Grundlagen Math. 5 (1959), 216-239.

\section{Massachusetts Institute of Technology, Cambridge, Massachusetts}

